\title{
A Network Pharmacology-Based Study of the Mechanism of Renshen Baidu Powder in Treating Ulcerative Colitis
}

Bo Jia ( $\sim$ Jiabochengdutcm@163.com )

Chengdu University of Traditional Chinese Medicine

\section{Xi-Yue Tan}

Chengdu University of Traditional Chinese Medicine

Xing-Long Liu

Chengdu University of Traditional Chinese Medicine

Xin-Yun Li

Chengdu University of Traditional Chinese Medicine

\section{Research Article}

Keywords: Inflammatory Bowel Disease, Crossover Genes Enrichment Analysis, Protein-protein Interaction, Gene Ontology, Kyoto Encyclopedia of Genes and Genomes, Anti-inflammatory Activities

Posted Date: February 17th, 2021

DOI: https://doi.org/10.21203/rs.3.rs-199959/v1

License: (a) (i) This work is licensed under a Creative Commons Attribution 4.0 International License. Read Full License 


\section{Abstract}

Ulcerative colitis (UC), one forms of chronic inflammatory bowel disease (IBD), is characterized by recurrent inflammation, and Renshen Baidu Powder (RSBDP) is often used as a traditional Chinese medicine prescription for treating of UC, but the pharmacological mechanisms remains unclear. This research aims to explore the mechanisms of RSBDP in the treatment of UC based on network pharmacology. Through multiple databases collected the compounds and targets, and constructed the network. The crossover genes enrichment analysis were performed by protein-protein interaction (PPI) construction and Gene Ontology (GO) and Kyoto Encyclopedia of Genes and Genomes (KEGG). Finally, 181 compounds and 266 targets were obtained. Potential candidate ingredients mainly include phytosterols, flavonoids, and coumarins, all of which have anti-inflammatory activities. GO and KEGG enrichment analysis suggested that RSBDP have anti-inflammatory and immunomodulatory effects. The effect of RSBDP on UC might be achieved by regulating the balance of cytokines (i.e., IL-6, TNF, IL-1) and the downstream mediators (i.e., STAT1, STAT3) in the immune system. And inflammation-, immune- and hypoxic-related pathways, like TNF, Toll-like receptors and HIF-1 signaling pathway. These results provide a theoretical basis for studying the effective substances of RSBDP in the treatment of UC and their mechanism of action for further research.

\section{Introduction}

Ulcerative colitis (UC) is a chronic inflammatory disease with pathological manifestations in the rectum and colon. The clinical manifestations are repeated abdominal pain, diarrhea, mucus pus and bloody stools. At the turn of the 21 st century, ulcerative colitis has become a global disease. Ulcerative colitis is a leading cause of morbidity and prevalence trends in Asia, the Middle East and South America increased, but it has stabilized in Western Europe, North America and Australia. The global risk of UC indicates the importance of environmental factors, immune response, microbial flora and genetic susceptibility. The exact cause of UC is remain clarified. It is believed to be closely associated with epithelial cells (colonocytes), mucous barrier and epithelial barrier defects ${ }^{1-5}$.

Besides, patients with long-standing IBD, especially UC, have an increased risk of developing colorectal cancer (CRC), which is one of the most critical complications ${ }^{6}$. According to reports, the incidence of CRC in IBD patients (i.e., colitis-associated cancer, CAC) was $60 \%$ higher than the general population ${ }^{7}$. Eaden's meta-analysis based on population cohort studies has shown that the overall prevalence of CRC in UC patients was $3.7 \%$. The incidence rate is equivalent to a cumulative probability of $2 \%$ at 10 years, $8 \%$ at 20 years, and $18 \%$ at 30 years $^{8}$. Bopanna et al. systematic review and meta-analysis have shown that the overall prevalence of CRC in Asia UC patients was $0.85 \%$. The cumulative risks of $0.02 \%, 4.81 \%$, and $13.91 \%$ at 10 years, 20 years, and 30 years $^{9}$. These results showed that the connection between the occurrence and development of inflammation and cancer is inevitable and intertwined, and inflammatory lesions may increase the risk of cancer occurrence and development. Therefore, it is necessary to reduce the risk of these patients suffering from colorectal cancer. 
The aim of UC management is to induce and maintain partial remission and improve the lives of patients. Current treatments for mild to moderate UC mainly contains 5-aminosalicylic acid (5-ASA) and corticosteroids (budesonide) 2,10-11. However, these therapies have low efficacy, and even with drug treatment, patients have a high short-term recurrence rate. Besides, there are many different management drugs for moderate to severe UC, including anti-TNF-a drugs (infliximab, adalimumab and golimumab), immunomodulators (thiopurines, methotrexate) and biological drugs ${ }^{2,12}$. Moreover, these therapies are accompanied by toxic side effects and have individual differences, and high financial costs are a barrier for patients. Thus, the current main task is to seek a more majorization and comprehensive treatment method.

So far, several studies have shown that traditional Chinese medicine (TCM) can treat UC, with low toxic side effects and easy acceptance by patients ${ }^{13-15}$. Renshen Baidu Powder(RSBDP), which originated in the Tang dynasty, is a famous Chinese medicine prescription that has been used for nearly 1400 years. The Chinese medicine preparation of RSBDP composed of ten TCMs: Panax ginseng C. A. Mey. (Renshen in Chinese), Notopterygii Rhizoma Et Radix (Qianghuo in Chinese), Radix Angelicae Biseratae (Duhuo in Chinese), Radix Bupleuri (Chaihu in Chinese), Platycodon Grandiforus (Jiegeng in Chinese), Aurantii Fructus (Zhike in Chinese), Chuanxiong Rhizoma (Chuanxiong in Chinese), Peucedani Radix (Qianhu in Chinese), Poria Cocos(Schw.) Wolf. (Fuling in Chinese), and licorice (Gancao in Chinese). Many clinical studies have suggested that RSBDP is used to treat UC across the Chinese Mainland ${ }^{16-17}$. Also, many experiments have shown that RSBDP can promote gastrointestinal motility function, improve the immune function of the body, repair intestinal mucosal damage ${ }^{18}$.

Network pharmacology is an emerging method that combines pharmacology, bioinformatics, chemoinformatics and systems biology. Nowadays, the scope of network pharmacology is expanding, including the study of the preparation of TCM and the exploration of drugs and diseases. TCM formula is a multi-target, multi-pathway, and multi-link system. Network pharmacology methods have been used to analyze the complexity between biological systems, drugs and diseases ${ }^{19-20}$. This study illustrates the mechanism of action of RSBDP in the treatment of UC from the perspective of network pharmacology. A flowchart of the study design is illustrated in Fig. 1.

\section{Materials And Methods}

Screening of the active compounds in RSBDP. The active compounds in RSBDP have been researched and collected from the TCM Systems Pharmacology Database (TCMSP, http://tcmspw.com/tcmsp.php), which is a useful tool based on systematic pharmacology for Chinese herbal medicines, including TCM compounds, targets, diseases and ADME-related properties ${ }^{21}$. ADME includes absorption, distribution, metabolism and excretion, and is an indispensable step in determining the pharmacokinetics of candidate drugs ${ }^{22}$. Among them, oral bioavailability (OB) and drug-like degree (DL) were selected as pharmacokinetic parameters. $\mathrm{OB}$, the oral absorption and permeability through pharmacological activity, is usually used to evaluate the development of drug candidates ${ }^{22-23}$. DL, the qualitative properties of 
chemicals, is widely used in the early stages of drug discovery and can predict drug-like molecules ${ }^{24-25}$. According to recent literature, $\mathrm{OB} \geq 30 \%$ and $\mathrm{DL} \geq 0.18$ are essential indicators for screening active compounds.

Targets Prediction. For the large number of the active compounds obtained above, we also summarized the relevant targets of RSBDP from TCMSP database. Besides, the UC-related targets come from multisource databases, including DrugBank database (https://go.drugbank.com//26, Therapeutic Target Database (TTD, http://bidd.nus.edu.sg/group/cjttd/) ${ }^{27}$, Online Mendelian Inheritance in Man database (OMIM, https://www.omim.org/) ${ }^{28}$, DisGeNET database (https://www.disgenet.org/) ${ }^{29}$, and using "ulcerative colitis" as a keyword to obtained disease targets. Then, the intersection of compoundtarget network and UC-related target network was integrated by the Venny 2.1.0 software (https://bioinfogp.cnb.csic.es/tools/venny/index.html) ${ }^{30}$. Finally, the candidate targets were mapped to the UniProt database (http://www.uniprot.org//31, and duplicate, and non-corresponding genes were eliminated.

Protein-protein interaction (PPI) Data. The data of PPI have come from the String database (https://string-db.org/cgi/input.pl) ${ }^{32}$, which is a database for the exploration and analysis of the proteinprotein association, including direct physical and indirect functional interactions between proteins. We set the organism as "Homo sapiens (human)," set the required interaction score at "high confidence (0.7)," and build a PPI network.

GO and KEGG Pathway Enrichment Analyses. GO biological function and KEGG pathway enrichment analysis were performed by The Database for Annotation, Visualization, and Integrated Discovery database (DAVID, https://david.ncifcrf.gov/) ${ }^{33}$, a web-based portal that provides gene list annotation and analysis resource. We set the genetic species to "Homo sapiens." And the Bar and Bubble Charts were plotted using the bioinformatics platform (http://www.bioinformatics.com.cn/) ${ }^{34}$.

Network Construction. Network construction was performed by Cytoscape 3.7.1 (http://www. cytoscape.org/ $)^{35}$, which is an open software project used to integrate the interaction network and status of biomolecules. Each node represents a compound, target or pathway, and edge indicates the interaction between nodes. Then, the following networks were constructed, including Herbs-Compounds network $(\mathrm{H}-\mathrm{C}$ networks), Compounds-Targets network (C-T network), PPI core targets network, and Target-Pathway network (T-P network).

\section{Results}

Potential Active Compounds and Herb-Compound network. A total of 1546 compounds of RSBDP were retrieved from TCMSP. Then, the following criteria were used for screening: $O B \geq 30 \%$ and $D L \geq 0.18$, and deleted the duplicate compounds, a total of 181 active compounds were obtained as a result.(Table 1). We was constructed the $\mathrm{H}-\mathrm{C}$ network by Cytoscape 3.7.1, and it contained 169 nodes (including 10 herbs and 159 compounds) and 181 edges (Fig. 2). Among 159 compounds, 12 of them provided a high degree 
(degree $\geq 2$ ). According to the descending order of degree, the compounds were beta-sitosterol (MOL000358, Degree=5), Sitosterol (MOL000359, Degree=4), quercetin (MOL000098, Degree=3), kaempferol (MOL000422, Degree=3), Imperatorin (MOL001941, Degree=3), isoimperatorin (MOL001942, Degree=3), nodakenin (MOL004792, Degree=3), isorhamnetin (MOL000354, Degree=2), Stigmasterol (MOL000449, Degree=2), Phellopterin (MOL002644, Degree=2), naringenin (MOL004328, Degree=2), (+)Anomalin (MOL004653, Degree=2).

Targets Prediction and Compound-Target Network. In this progress, a total of 266 predicted targets of 181 active compounds were obtained. Then, we have integrated various disease gene databases, including DrugBank, TTD, OMIM, DisGeNET, and obtained 857 UC-related targets. Finally, the collected UC targets and RSBDP targets were intersected, and a total of 92 RSBDP-UC targets were obtained(Fig. 3a,b).

Based on the above research, the RSBDP C-T network was constructed by Cytoscape 3.7.1, which contained 233 nodes(including 141 compounds and 92 RSBDP-UC targets), and 1145 edges (Fig. 4). These network show that multiple compounds can regulate targets, and indicate the active compounds and the targets interaction.

PPI Network analysis. To further obtain the PPI network, 92 RSBDP-UC targets were input into the String database, and removed the unconnected nodes. Then, the result was imported into Cytoscape3.7.1, and obtained the network which contained 85 nodes and 704 edges (Fig. 5). To analyze the core PPI network, we were screened out with the following topological characteristics, including " Degree Centrality(DC) $\geq$ 20, Closeness Centrality (CC) $\geq 0.48$ and Betweenness Centrality $(B C) \geq 0$ ". Finally, we were selected the top 30 targets of the PPI network (Table. 1), and obtained 30 nodes and 318 edges (Fig. 6a,b).

GO Biological Processes (BP) enrichment analysis. In order to further identify whether the enrichment of the biological processes of the above candidate targets were related to UC. GO-BP enrichment analysis was performed by DAVID, and we were obtained the top 20 significant GO BPs(Fig. 7). GO BP terms are mainly enriched in inflammatory response, regulation of apoptotic process and regulation of transcription, such as "inflammatory response," "negative regulation of apoptotic process," "aging," "positive regulation of transcription, DNA-templated" and "positive regulation of transcription from RNA polymerase II promoter."(Table. 2).

KEGG pathway enrichment analysis. In this progress, KEGG pathway enrichment analysis was used to further reveal the mechanism of UC remission. The pathways related to UC mainly included TNF signaling pathway (hsa04668), Toll-like receptors signaling pathway (hsa04620), HIF-1 signaling pathway (hsa04066), NOD-like receptors signaling pathway (hsa04621), T-cell receptor signaling pathway (hsa04660) and so on (Fig.8). In addition, a T-P network was constructed according to the target of RSBDP on each pathway (Fig. 9).

\section{Discussion}


TCM has the advantages of reducing side effects, improving therapeutic efficacy, and have low treatment cost. Nowadays, network pharmacology provides a new approach to study TCM formulas and the advantages of multi-compound, multi-target and multi-pathway characteristics of TCM formula.

Based on the ADEM properties, we have screened out 181 active compounds of RSBDP. Then, the H-T network of RSBDP had 159 active compounds, including $\beta$-sitosterol, Sitosterol, quercetin, kaempferol, Imperatorin, isoimperatorin, nodakenin, isorhamnetin, Stigmasterol, Phellopterin, naringenin, (+)Anomalin, and the compounds as mentioned above can act on 2-5 Chinese herbal medicines simultaneously, and were divided into three major categories, including Phytosterols, Flavonoids and Coumarins. Phytosterols, the plant analogs of cholesterol, including $\beta$-sitosterol, Sitosterol and Stigmasterol. Recently, many studies have shown that phytosterols have anti-inflammatory functions ${ }^{36-}$ 37. $\beta$-sitosterol has shown many biological functions, including lowing cholesterol level, anti-inflammatory and anticancer effects. Oral $\beta$-sitosterol $(20 \mathrm{mg} / \mathrm{kg})$ may significantly inhibit the expression of cytokines TNF- $\alpha$, IL-1 $\beta$, and IL- 6 in the colon of male C57BL/ 6 mice with TNBS-induced colitis and simultaneously inhibits the expression of cyclooxygenase (COX)-2 and the phosphorylation of $p 65^{38}$. $\beta$-sitosterol can inhibit the activation of NF-KB by inhibiting the binding between lipopolysaccharides (LPS) and toll-like receptor (TLR) 4 to express anti-inflammatory effects ${ }^{39}$. Furthermore, Feng et al. studies have shown that dietary phytosterols, $\beta$-sitosterol and stigmasterol inhibited NF-KB activation in mice with DSS-induced colitis $^{40}$. Flavonoids are widely distributed in the plant kingdom, including quercetin, kaempferol, isorhamnetin and naringenin. Quercetin has multiple biological functions, like anti-inflammatory, anticancer, antioxidant and anti-hypertensive. Quercetin regulates epithelial inflammatory genes associated with LPS-induced models, which down-regulated C/EBP- $\beta$, and Lcn-2, and up-regulated Hmox 1 , and Fpn $1^{41}$. The most important properties of kaempferol are anti-inflammatory, can be used to treat numerous diseases caused by acute and chronic inflammation. Park et al. have been confirmed that kaempferol down-regulated the colonic mRNA expression levels of the inflammatory cytokines (i.e., TNF$a$, IL-1 $\beta$, and IL-6) in the colons of female C57BL/6J mice with DSS-induced colitic and inhibited the activation of myeloperoxidase (MPO) ${ }^{42}$. Coumarins are plant-derived products, including Imperatorin, isoimperatorin, nodakenin, Phellopterin and (+)-Anomalin. The antioxidant, anticancer and antiinflammatory effects have been recognized in many in vivo and in vitro studies ${ }^{43-44}$. Imperatorin (IMP) can effectively reduce the increase of IL-1 $\beta$ and TNF-a levels in LPS-induced mice ${ }^{45}$. Luo et al. reported that IMP inhibited TNF- $a$ and IL-6 in TNBS-induced colitis mice and alleviated the symptoms of UC by regulating the Nrf-2/ARE/HO-1 pathway ${ }^{46}$.

According to the active compounds obtained above and matched with related UC targets, we were obtained 92 RSBDP-UC targets, and constructed the C-T network and PPI network. Cytokines such as TNF, IL-1, IL-6, IFN- $y$, IL-2, and IL-8 (CXCL8) were produced by Th1 cells, which mediate cellular immune responses to pathogens and participate in promoting the development of CRC and CAC. In contrast, IL-4 and IL-10 were produced by Th2 cells, which are related to anti-inflammatory response ${ }^{47}$. If the balance between Th1 and Th2 is lost, inflammation of the tissues and viscera will occur, leading to the occurrence and development of diseases. TNF is a well-known pro-inflammatory cytokine, which is mainly secreted 
by monocytes or macrophages. Signaling pathways and transcription factors (i.e., NF-kB) were triggered by the activation of TNF-a ${ }^{48-49}$. Like TNF-a, IL-1 plays a crucial signal in the initial alarm, causing a series of up-regulation and anti-inflammatory responses to be activated. Among the IL-1 family, IL-1a and IL-1 $\beta$ are cytokines that have been widely studied. And evidence suggested that IL-1 $\beta$ can indirectly activate endothelial cells and angiogenesis by up-regulating pro-inflammatory effects and pro-angiogenic molecules. Moreover, the findings have shown that the activation blockage of IL-1 $\beta$ reduces tumorigenesis in a murine CAC model by impairing macrophage-dependent IL- 6 secretion. These findings suggested that IL-1 has a significant effect on the initiation of colonic inflammation ${ }^{50-51}$.

IL-6 is essential for long-standing inflammation. It is used to inhibits the effect of regulatory T cells, and involved in activating STAT3, a major oncogenic transcription factor. STAT1 and STAT3 as a downstream targets of IL- 6 signaling, which can prolong the survival period of CRC patients. The inhibition of colitis activity is related to the inhibition of STAT3 activation, the induction of T cell apoptosis and the reduction of pro-inflammatory cytokines ${ }^{49-50,52-53}$. Gordziel et al. have shown that the expression of STAT1 and STAT3 are correlated respectively with IL-6, and the significantly expression of IL-6 in CRC biopsies tissues are both shown to a tendency for association with longer survival ${ }^{55}$.

Then, the T-P network were constructed and performed enrichment analysis, and the results reflected that 85 targets could be mapped to 179 pathways. Five pathways of the top 20 pathways, including "TNF signaling pathway," "Toll-like receptors signaling pathway," "NOD-like receptors signaling pathway," and "HIF-1 signaling pathway," were closely related to inflammatory and immune function. TNF signaling pathway acts as an important role in a regulator of inflammation. TNF has two primary receptors, such as TNF receptors 1 and 2(TNFR1 and TNFR2). In UC, high tissue levels of TNF and cytokines (i.e., IFN- $\gamma$, IL-17 and IL-12) can cause a series of symptoms such as diarrhea. Bradford et al. studies have shown that TNF promotes the recovery of colitis mucosa by acting on the colonic epithelial stem and progenitor cell populations ${ }^{55}$. Pattern recognition receptors (PRPs) are essential for regulating the homeostasis of the intestinal immune system and are mediators of inflammation in the gastrointestinal tract, including Toll-like receptors (TLRs), NOD-like receptors (NLRs) and RIG-like receptors (RLR) ${ }^{56}$. TLRs signaling pathway mainly control immune function and can act as a risk modifier for UC and CAC. It has been found that TLRs expressed in the intestinal epithelium are related to the long-term existence of mucosal inflammation, result in a high degree of morbidity. Activation of the TLR signal transduction pathway induces pro-inflammatory cytokines, and transcription factors like NF-KB to target invaders ${ }^{57}$. Once activated, TLRs trigger the subsequent activation of downstream signaling cascades and induce the activation of NF-KB. TLR mutations and dysregulation are the main factors in the predisposition and susceptibility to $\mathrm{IBD}^{58-59}$. NLRs signaling pathways are involved in the regulation of inflammatory and apoptotic responses. A pivotal role of NOD2 in the inhibition of intestinal inflammation and tumorigenesis. Kurzawski et al. reported the NOD2 might be a risk factor for $\mathrm{CRC}^{60}$. Udden et al. studies in vivo have shown that NOD2-mediated protection against colorectal tumorigenesis, and the inhibition of TLR-mediated activation of NF-KB pathways and MAPK pathway, and promotion of the inflammatory cytokines $^{61}$. 
However, recent studies have provided evidence that a comparison of chronic inflammation and normal tissues found that hypoxic mainly exists in the former. The adaptive responses to hypoxic are mediated via the Hypoxia Inducible transcription Factors $(\mathrm{HIFs})^{62}$. HIF-1 signaling pathway has been shown to have a protective effect in colitis in vivo. The HIF pathway is regulated by a family of oxygen-sensitive HIF prolyl hydroxylases. The expression and transcriptional activity of HIF-1 was inhibited by hydroxylases ${ }^{62-}$

63. Cummins et al. studies in vivo and in vitro have demonstrated that the pan-hydroxylase inhibitor, dimethyloxalylglycine (DMOG), can induce the activation of HIF-1 in cultured intestinal epithelial cells, and accelerate recovery in DSS-induced female C57BL/6 mice, and promote the pro-inflammation cytokines in DMOG-treated mice ${ }^{64}$.

These results reveal that RSBDP not only can regulate pro- and anti-inflammatory, immune and antihypoxic effects but also can prevent the occurrence and development of tumors. Moreover, the occurrence and development of UC were related to the imbalance of cytokines and downstream mediators. These networks in this study illustrate the features of RSBDP multi-compounds, multi-targets and multi-pathways therapy of UC with the complicated and varied action mechanisms.

\section{Conclusion}

In summary, we have identified 181 active compounds that mediate 85 UC-related targets to play a synergistic effect. The multiple signal pathways and biological processes can enrich same target, indicating the prescription has a multi-target and multi-pathway synergistic effect on UC. At the same time, this research remain has limitations, one of them is based on the prediction results obtained from existing databases. Simultaneously, this research only provided a preliminary hypothesis for scientific research in TCM, which requires systematic experimental research to further confirm.

\section{Declarations}

\section{Acknowledgements}

This study was supported by the National Natural Science Foundation of China (No. 325020015).

\section{Author contributions}

X.Y.T. wrote the manuscript; X.Y.T. and X.L.L. edited and improved the manuscript; X.Y.L. were responsible for coordinating this study. X.L.L. and B.J. directed the research and proposed changes to the manuscript, and all authors reviewed the manuscript and approved the final version of the manuscript.

\section{Additional Information}

Competing interests The authors declare no competing interests

Correspondence and requests for materials should be addressed to X.Y.T. 
Reprints and permissions information is available at www.nature.com/reprints.

\section{References}

1. Ng SC, Shi HY, Hamidi $\mathrm{N}$, et al. Worldwide incidence and prevalence of inflammatory bowel disease in the 21st century: a systematic review of population-based studies. Lancet. 390, 2769-2778(2018).

2. Ungaro R, Mehandru S, Allen PB, Peyrin-Biroulet L, Colombel JF. Ulcerative colitis. Lancet. 389, 17561770(2017).

3. Matsuoka K, Kobayashi T, Ueno F, et al. Evidence-based clinical practice guidelines for inflammatory bowel disease. J Gastroenterol. 53, 305-353(2018).

4. Zhang YZ, Li YY. Inflammatory bowel disease: pathogenesis. World J Gastroenterol. 20, 91-99(2014).

5. Fumery M, Singh S, Dulai PS, et al. Natural History of Adult Ulcerative Colitis in Population-based Cohorts: A Systematic Review. Clin Gastroenterol Hepatol. 16, 343-356(2018).

6. Lukas M. Inflammatory bowel disease as a risk factor for colorectal cancer. Dig Dis. 28, 619624(2010).

7. Long AG, Lundsmith ET, Hamilton KE. Inflammation and Colorectal Cancer. Curr Colorectal Cancer Rep. 13, 341-351(2017).

8. Eaden JA, Abrams KR, Mayberry JF. The risk of colorectal cancer in ulcerative colitis: a metaanalysis. Gut. 48, 526-535(2001).

9. Bopanna S, Ananthakrishnan AN, Kedia S, Yajnik V, Ahuja V. Risk of colorectal cancer in Asian patients with ulcerative colitis: a systematic review and meta-analysis. Lancet Gastroenterol Hepatol. 2, 269-276(2017).

10. Ben-Horin S, Andrews JM, Katsanos KH, et al. Combination of corticosteroids and 5-aminosalicylates or corticosteroids alone for patients with moderate-severe active ulcerative colitis: A global survey of physicians' practice. World J Gastroenterol. 23, 2995-3002(2017).

11. Ko CW, Singh S, Feuerstein JD, et al. AGA Clinical Practice Guidelines on the Management of Mild-toModerate Ulcerative Colitis. 156, 748-764(2019).

12. Feuerstein JD, Isaacs KL, Schneider Y, et al. AGA Clinical Practice Guidelines on the Management of Moderate to Severe Ulcerative Colitis. Gastroenterology. 158, 1450-1461(2020).

13. Gu S, Xue Y, Zhang Y, et al. An Investigation of the Mechanism of Rapid Relief of Ulcerative Colitis Induced by Five-flavor Sophora Flavescens Enteric-coated Capsules Based on Network Pharmacology. Comb Chem High Throughput Screen. 23, 239-252(2020).

14. Chen, You-Lan et al. Systems pharmacology approach reveals protective mechanisms of Jian-Pi Qing-Chang decoction on ulcerative colitis. World journal of gastroenterology. 25, 2603-2622(2019): .

15. Ji, Jun et al. Review of Clinical Studies of the Treatment of Ulcerative Colitis Using Acupuncture and Moxibustion. Gastroenterology research and practice. 2016, 9248589(2016).

16. Li JY.Treatment of 49 Cases of Ulcerative Colitis with the Method of Ni liu wan zhou. JOURNAL OF PRACTICAL TRADITIONAL CHINESE INTERNAL MEDICINE, 18, 342-343(2004). 
17. Liu XL, Huang XS, Jia B, et al. A Brief Discussion on the Application of Ni liu wan zhou in Remission of Ulcerative Colitis. Journal of Sichuan of Traditional Chinese Medicine, 33, 21-23(2015).

18. Chen L, Jia B, et al. Exploring the effect of Renshen Baidu Powder on gastrointestinal function in rats with ulcerative colitis and its mechanism based on the method of "Ni liu wan zhou". SHAANXI ZHONGYI, 40, 283-286(2019).

19. Zhou Z, Chen B, Chen S, et al. Applications of Network Pharmacology in Traditional Chinese Medicine Research. Evid Based Complement Alternat Med. 2020, 1646905(2020).

20. Zhang R, Zhu X, Bai H, Ning K. Network Pharmacology Databases for Traditional Chinese Medicine: Review and Assessment. Front Pharmacol. 10, 123(2019).

21. Ru J, Li P, Wang J, et al. TCMSP: a database of systems pharmacology for drug discovery from herbal medicines. J Cheminform. 6, 13(2014).

22. Xu X, Zhang W, Huang C, et al. A novel chemometric method for the prediction of human oral bioavailability. Int J Mol Sci. 13, 6964-6982(2012).

23. Ahmed SS, Ramakrishnan V. Systems biological approach of molecular descriptors connectivity: optimal descriptors for oral bioavailability prediction. PLoS One. 7, e40654(2012).

24. Li Y, Wang J, Lin F, Yang Y, Chen SS. A Methodology for Cancer Therapeutics by Systems Pharmacology-Based Analysis: A Case Study on Breast Cancer-Related Traditional Chinese Medicines. PLoS One. 12, e0169363(2017).

25. Tian S, Wang J, Li Y, et al. The application of in silico drug-likeness predictions in pharmaceutical research. Adv Drug Deliv Rev. 86, 2-10(2015).

26. Wishart DS, Feunang YD, Guo AC, et al. DrugBank 5.0: a major update to the DrugBank database for 2018. Nucleic Acids Res. 46, 1074-1082(2018).

27. Wang Y, Zhang S, Li F, et al. Therapeutic target database 2020: enriched resource for facilitating research and early development of targeted therapeutics. Nucleic Acids Res. 48, 1031-1041(2020).

28. Amberger JS, Bocchini CA, Schiettecatte F, Scott AF, Hamosh A. OMIM.org: Online Mendelian Inheritance in Man $\left(\mathrm{OMIM}{ }^{\circledR}\right)$, an online catalog of human genes and genetic disorders. Nucleic Acids Res. 43, 789-798(2015).

29. Piñero J, Bravo À, Queralt-Rosinach N, et al. DisGeNET: a comprehensive platform integrating information on human disease-associated genes and variants. Nucleic Acids Res. 45, 833839(2017).

30. Oliveros, J.C. Venny. An interactive tool for comparing lists with Venn's diagrams. https://bioinfogp.cnb.csic.es/tools/venny/index.html (2007-2015)

31. The UniProt Consortium, UniProt: a worldwide hub of protein knowledge, Nucleic Acids Research, 47, 506-515(2019).

32. Szklarczyk D, Gable AL, Lyon D, et al. STRING v11: protein-protein association networks with increased coverage, supporting functional discovery in genome-wide experimental datasets. Nucleic Acids Res. 47, 607-613(2019). 
33. Huang DW, Sherman BT, Lempicki RA. Systematic and integrative analysis of large gene lists using DAVID Bioinformatics Resources. Nature Protoc. 4, 44-57(2009).

34. Bioinformatics database, an online platform for data analysis and visualization. Heatmap was plotted by http://www.bioinformatics.com.cn (2020).

35. Shannon P, Markiel A, Ozier O, et al. Cytoscape: a software environment for integrated models of biomolecular interaction networks. Genome Res. 13, 2498-2504(2003).

36. Aldini R, Micucci M, Cevenini M, et al. Antiinflammatory effect of phytosterols in experimental murine colitis model: prevention, induction, remission study. PLoS One. 9, e108112(2014).

37. Othman RA, Moghadasian MH. Beyond cholesterol-lowering effects of plant sterols: clinical and experimental evidence of anti-inflammatory properties. Nutr Rev. 69, 371-382(2011).

38. Lee IA, Kim EJ, Kim DH. Inhibitory effect of $\beta$-sitosterol on TNBS-induced colitis in mice. Planta Med. 78, 896-898(2012).

39. Kim KA, Lee IA, Gu W, Hyam SR, Kim DH. $\beta$-Sitosterol attenuates high-fat diet-induced intestinal inflammation in mice by inhibiting the binding of lipopolysaccharide to toll-like receptor 4 in the NFKB pathway. Mol Nutr Food Res. 58, 963-972(2014).

40. Feng $S$, Dai Z, Liu A, et al. $\beta$-Sitosterol and stigmasterol ameliorate dextran sulfate sodium-induced colitis in mice fed a high fat Western-style diet. Food Funct. 8, 4179-4186(2017);(11).

41. Dicarlo M, Teti G, Verna G, et al. Quercetin Exposure Suppresses the Inflammatory Pathway in Intestinal Organoids from Winnie Mice. Int J Mol Sci. 20, 5771(2019).

42. Park MY, Ji GE, Sung MK. Dietary kaempferol suppresses inflammation of dextran sulfate sodiuminduced colitis in mice. Dig Dis Sci. 57, 355-363(2012).

43. Witaicenis A, Seito LN, da Silveira Chagas A, et al. Antioxidant and intestinal anti-inflammatory effects of plant-derived coumarin derivatives. Phytomedicine. 21, 240-246(2014).

44. Hassanein EHM, Sayed AM, Hussein OE, Mahmoud AM. Coumarins as Modulators of the Keap1/Nrf2/ARE Signaling Pathway. Oxid Med Cell Longev. 2020, 1675957(2020).

45. Singh G, Kaur J, Kaur M, Singh P, Bhatti R. Anti-nociceptive and anti-inflammatory effect of imperatorin: evidences for involvement of COX-2, iNOS, NFKB and inflammatory cytokines. Int $J$ Neurosci. 130, 176-185(2020).

46. Luo M, Luo Y. Imperatorin Relieved Ulcerative Colitis by Regulating the Nrf-2/ARE/HO-1 Pathway in Rats. Inflammation. 23, 1007/s10753-020-01353-3 (2020).

47. Gandhi GR, Neta MTSL, Sathiyabama RG, et al. Flavonoids as Th1/Th2 cytokines immunomodulators: A systematic review of studies on animal models. Phytomedicine. 44, 7484(2018).

48. Bradford EM, Ryu SH, Singh AP, et al. Epithelial TNF Receptor Signaling Promotes Mucosal Repair in Inflammatory Bowel Disease. J Immunol. 199, 1886-1897(2017).

49. Hnatyszyn A, Hryhorowicz S, Kaczmarek-Ryś M, et al. Colorectal carcinoma in the course of inflammatory bowel diseases. Hered Cancer Clin Pract. 17, 18(2019). 
50. Francescone R, Hou V, Grivennikov SI. Cytokines, IBD, and colitis-associated cancer. Inflamm Bowel Dis. 21, 409-418(2015).

51. Wang $Y$, Wang K, Han GC, et al. Neutrophil infiltration favors colitis-associated tumorigenesis by activating the interleukin-1 (IL-1)/IL-6 axis. Mucosal Immunol. 7, 1106-1115(2014).

52. Waldner MJ, Foersch S, Neurath MF. Interleukin-6-a key regulator of colorectal cancer development. Int J Biol Sci. 8, 1248-1253(2012).

53. Waldner MJ, Neurath MF. Master regulator of intestinal disease: IL-6 in chronic inflammation and cancer development. Semin Immunol. 26, 75-79(2014).

54. Gordziel C, Bratsch J, Moriggl R, Knösel T, Friedrich K. Both STAT1 and STAT3 are favourable prognostic determinants in colorectal carcinoma. Br J Cancer. 109, 138-146(2013).

55. Bradford EM, Ryu SH, Singh AP, et al. Epithelial TNF Receptor Signaling Promotes Mucosal Repair in Inflammatory Bowel Disease. J Immunol. 199, 1886-1897(2017).

56. Davis BK, Philipson C, Hontecillas R, et al. Emerging significance of NLRs in inflammatory bowel disease. Inflamm Bowel Dis. 20, 2412-2432(2014).

57. Vidya MK, Kumar VG, Sejian V, et al. Toll-like receptors: Significance, ligands, signaling pathways, and functions in mammals. Int Rev Immunol. 37, 20-36(2018).

58. Lu Y, Li X, Liu S, Zhang Y, Zhang D. Toll-like Receptors and Inflammatory Bowel Disease. Front Immunol. 9, 72(2018).

59. Kordjazy N, Haj-Mirzaian A, Haj-Mirzaian A, et al. Role of toll-like receptors in inflammatory bowel disease. Pharmacol Res. 129, 204-215(2018).

60. Kurzawski G, Suchy J, Kładny J, et al. The NOD2 3020insC mutation and the risk of colorectal cancer. Cancer Res. 64, 1604-1606(2004).

61. Udden SMN, Peng L, Gan JL, et al. NOD2 Suppresses Colorectal Tumorigenesis via Downregulation of the TLR Pathways. Cell Rep. 19, 2756-2770(2017).

62. Szade A, Grochot-Przeczek A, Florczyk U, Jozkowicz A, Dulak J. Cellular and molecular mechanisms of inflammation-induced angiogenesis. IUBMB Life. 67, 145-159(2015).

63. Karhausen J, Furuta GT, Tomaszewski JE, et al. Epithelial hypoxia-inducible factor-1 is protective in murine experimental colitis. J Clin Invest. 114, 1098-1106(2004).

64. Cummins EP, Seeballuck F, Keely SJ, et al. The hydroxylase inhibitor dimethyloxalylglycine is protective in a murine model of colitis. Gastroenterology. 134, 156-165(2008).

\section{Figures}




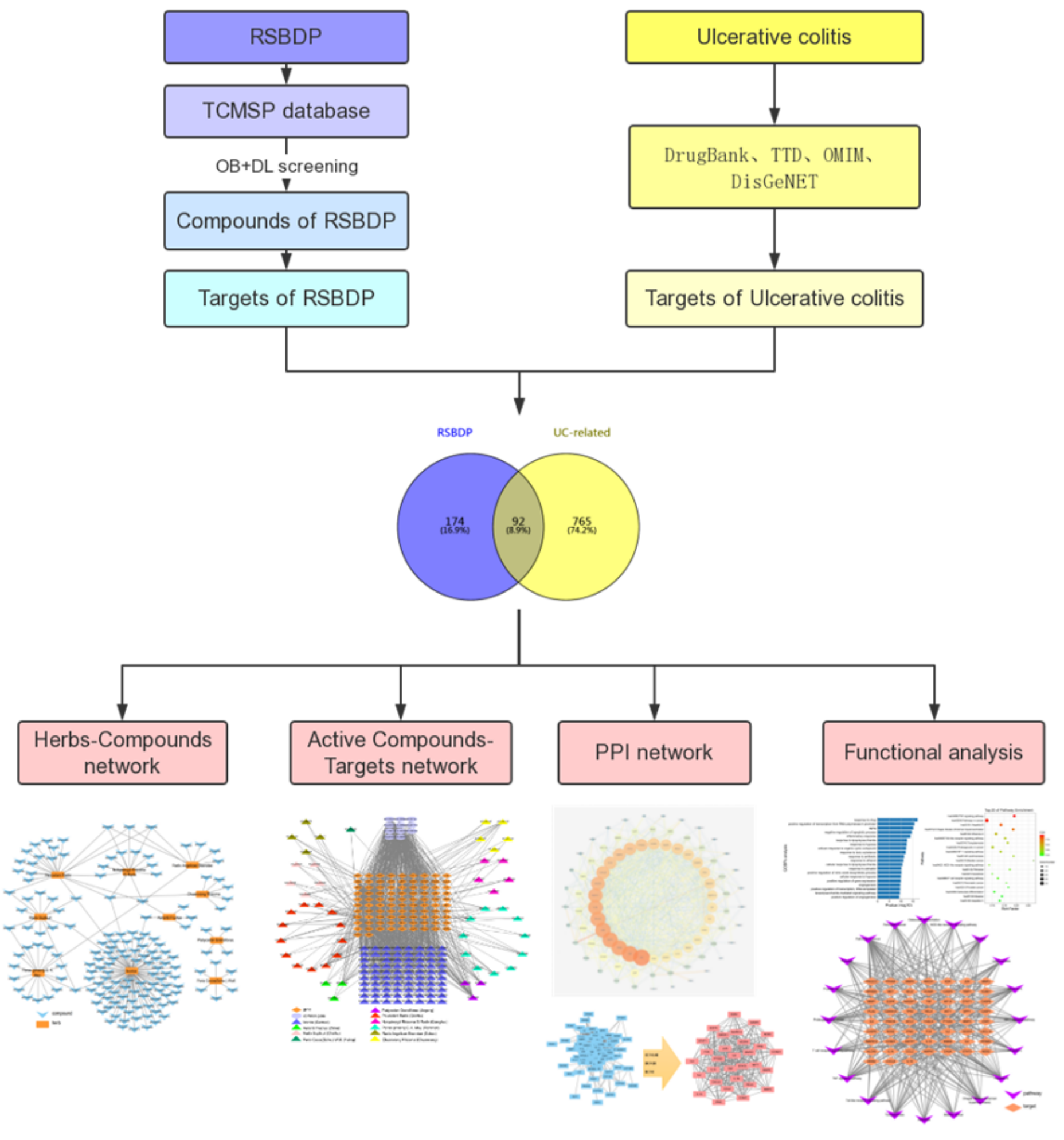

\section{Figure 1}

Framework of network pharmacological of RSBDP based on an integration strategy 


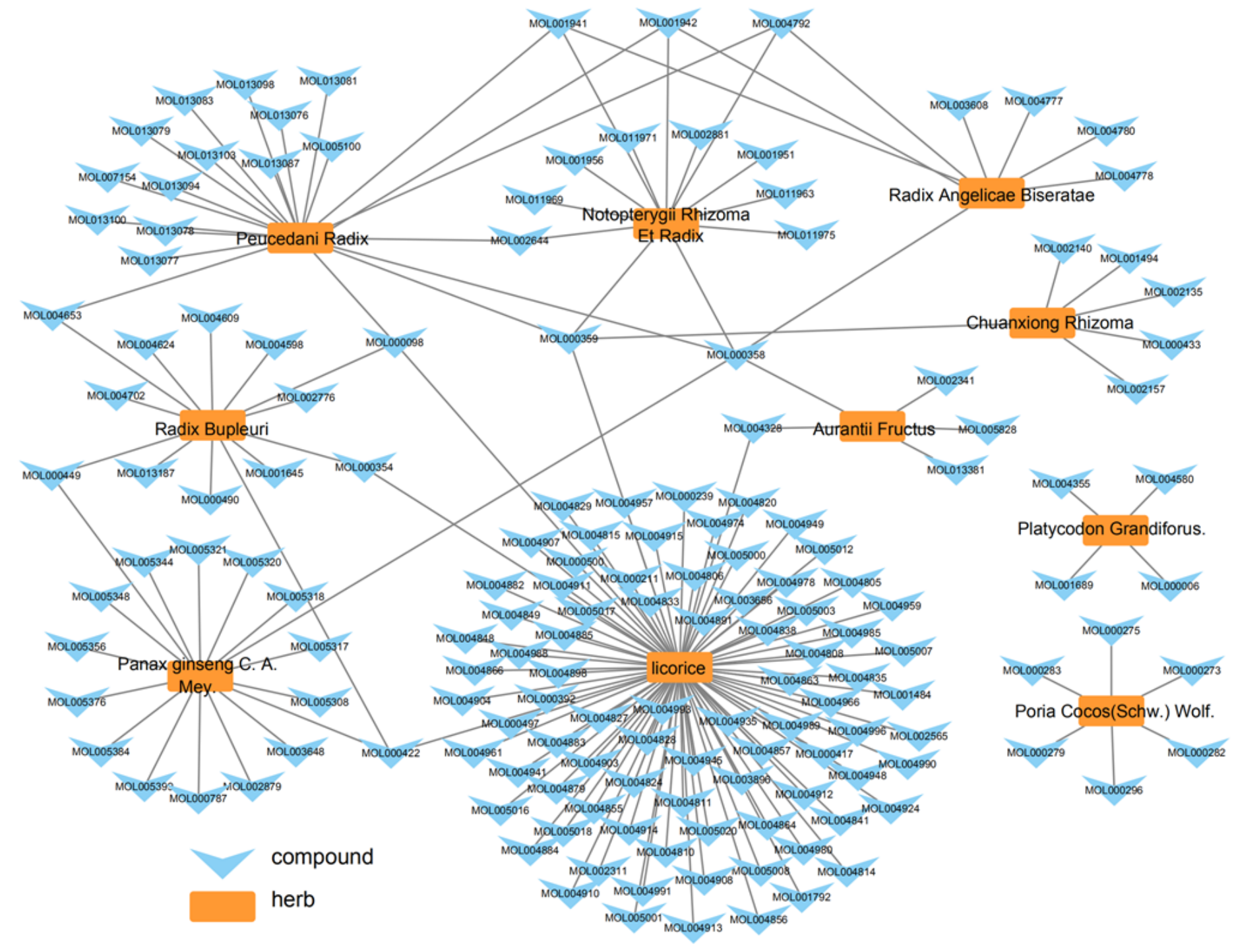

Figure 2

Herb-Compound network ( $\mathrm{H}-\mathrm{C}$ network). The edges represent the interaction between herb and compound. 


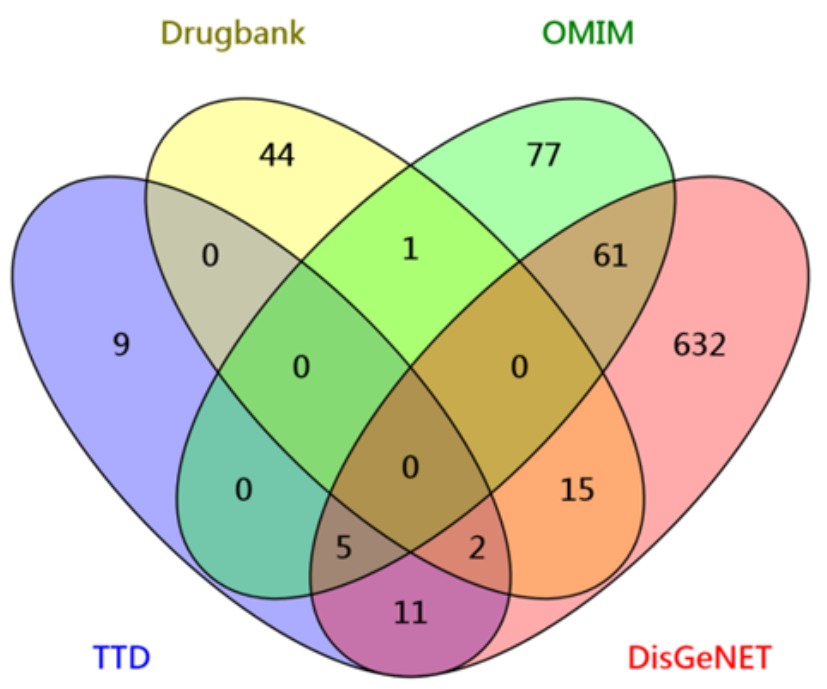

(a)

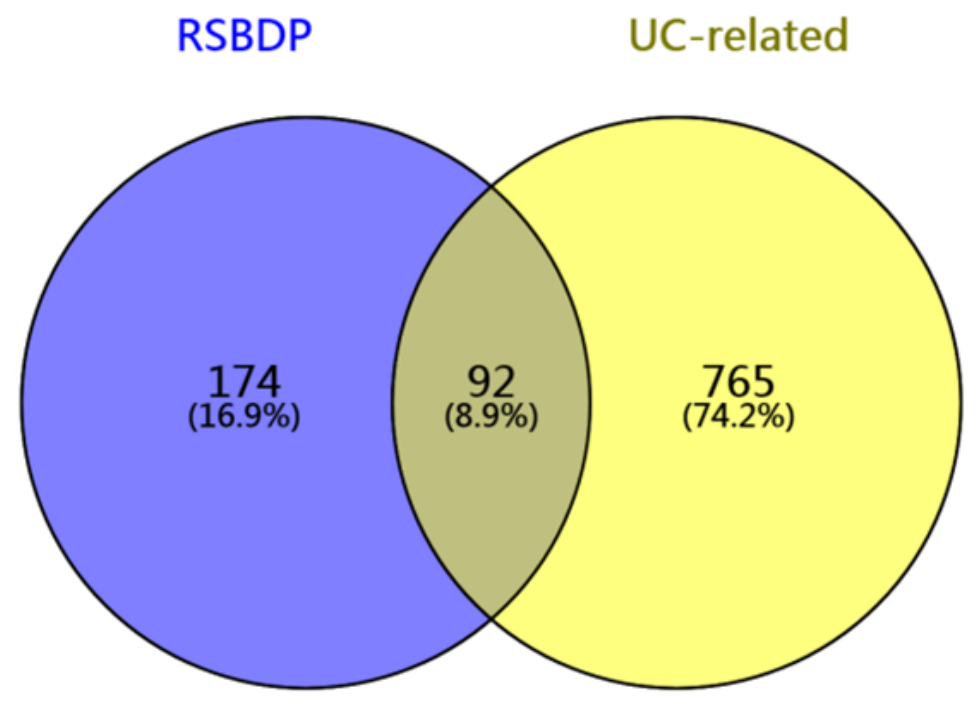

(b)

Figure 3

Venn diagram of the targets in UC and RSBDP. 


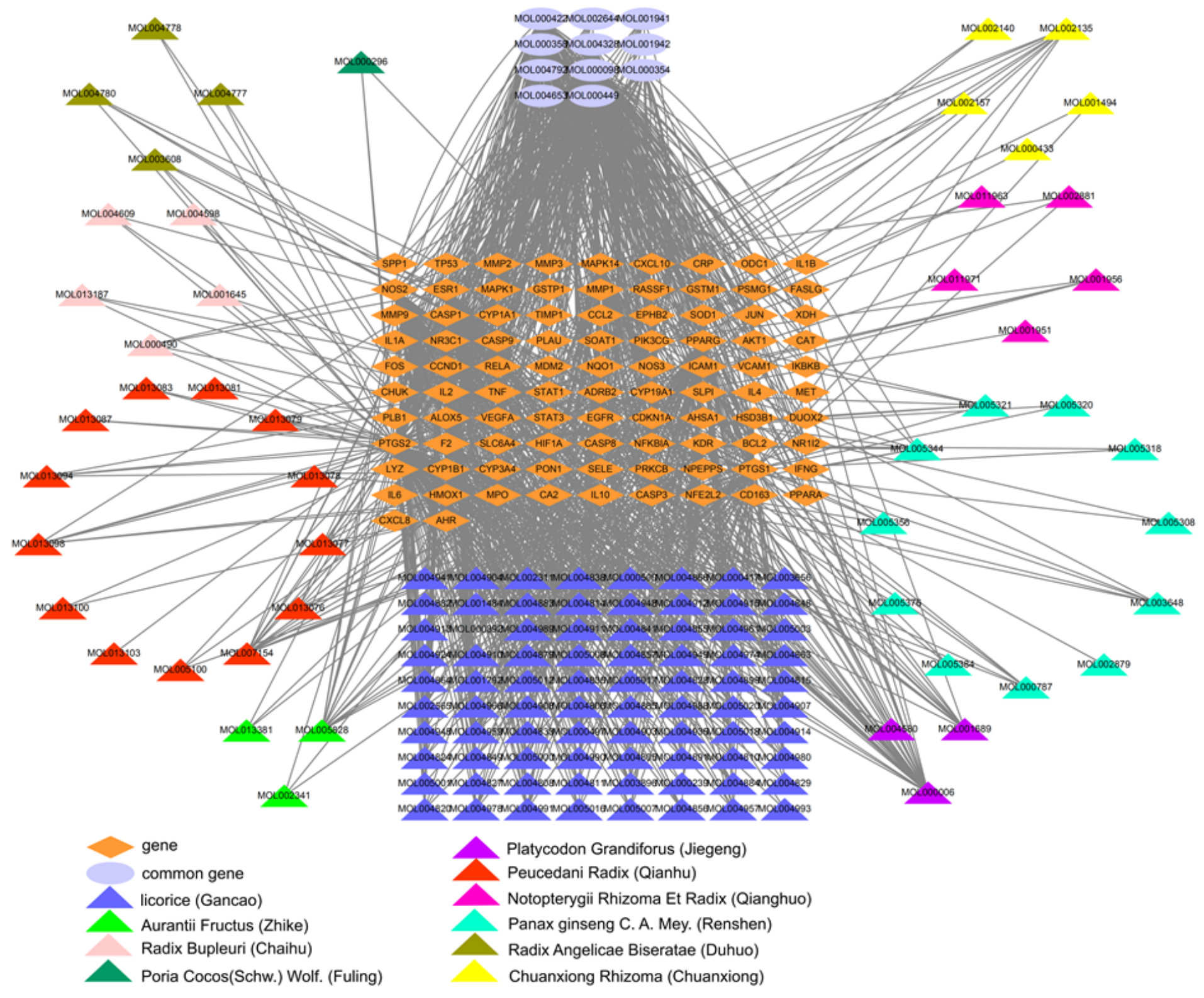

Figure 4

Compound-Targets network (C-T network) of RSBDP. 




Figure 5

The PPI network of 85 nodes. The size of node is proportional to degree. 


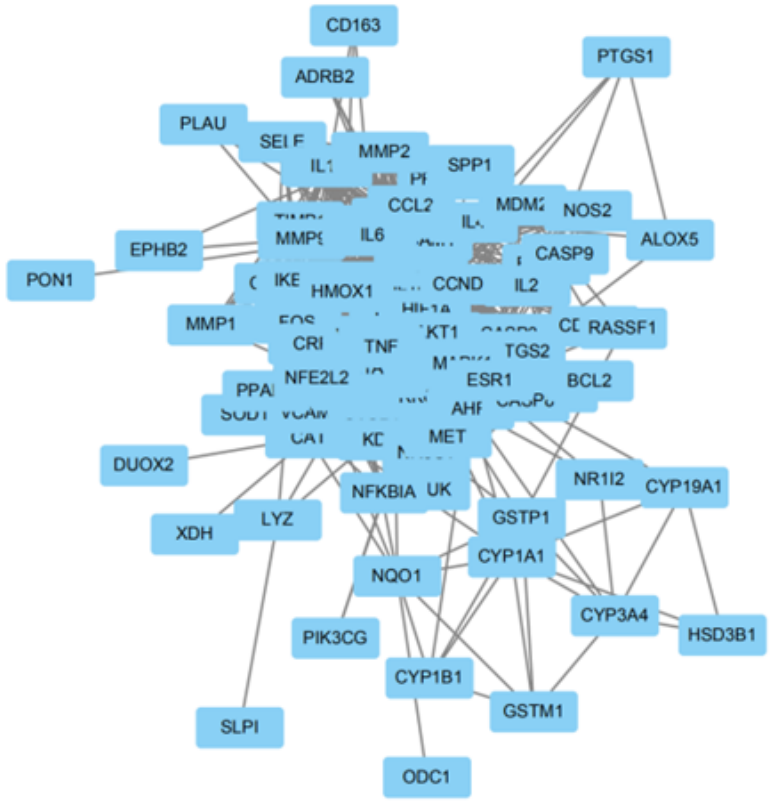

(a) 85 nodes and 704 edges

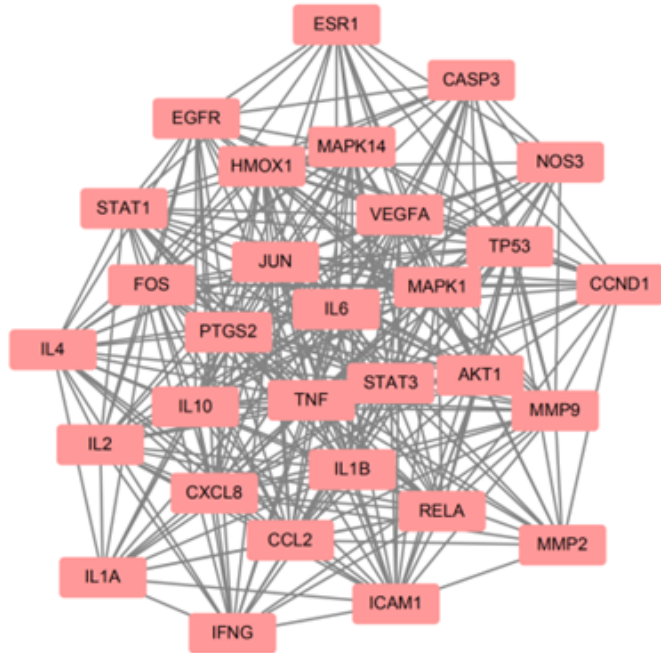

(b) 30 nodes and 318 edges

\section{Figure 6}

The progress of screening for the PPI network.

$\frac{\infty}{\infty}$
$\frac{1}{0}$
$\frac{1}{c}$
0
0
0
0
0 response to drug positive regulation of transcription from RNA polymerase II promoter aging negative regulation of apoptotic process inflammatory response response to lipopolysaccharide response to hypoxia cellular response to organic cyclic compound response to toxic substance response to antibiotic response to ethanol cellular response to lipopolysaccharide response to estradiol positive regulation of nitric oxide biosynthetic process cellular response to hypoxia positive regulation of gene expression angiogenesis positive regulation of transcription, DNA-templated lipopolysaccharide-mediated signaling pathway positive regulation of angiogenesis

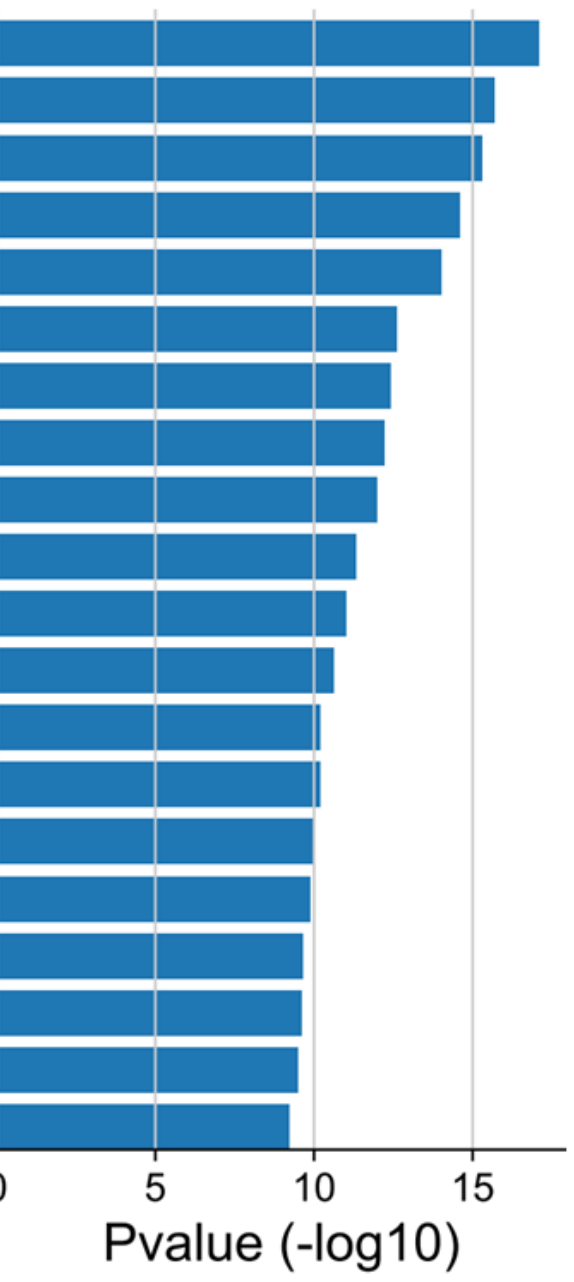




\section{Figure 7}

The GO BPs analysis of targets of RSBDP. Enrichment results were obtained for biological processes; only the top $20 \mathrm{GO}$ terms with $\mathrm{P}<0.05$ are displayed for each category.

\section{Top 20 of Pathway Enrichment}

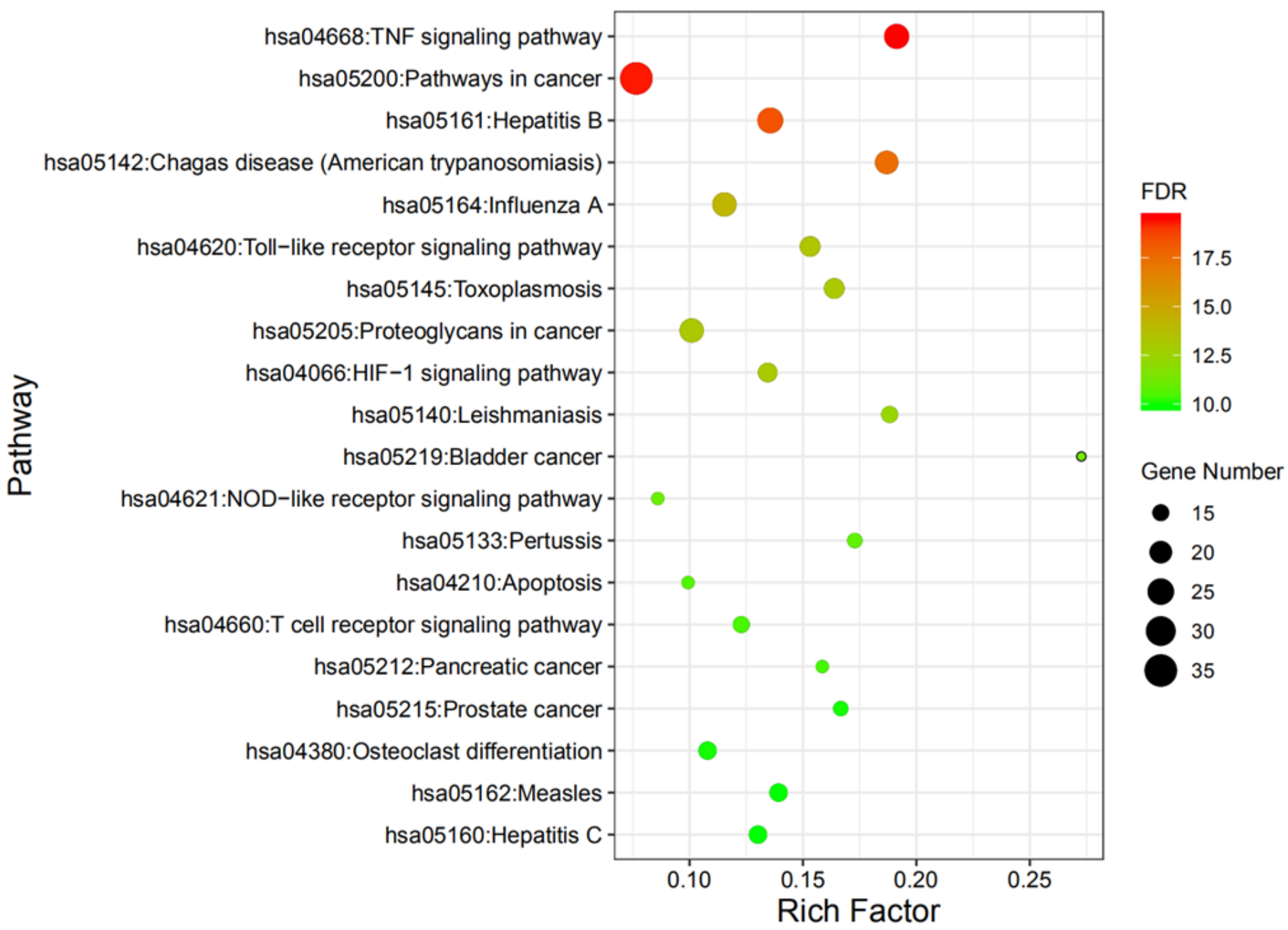

\section{Figure 8}

The top 20 KEGG enrichment pathway analysis. 


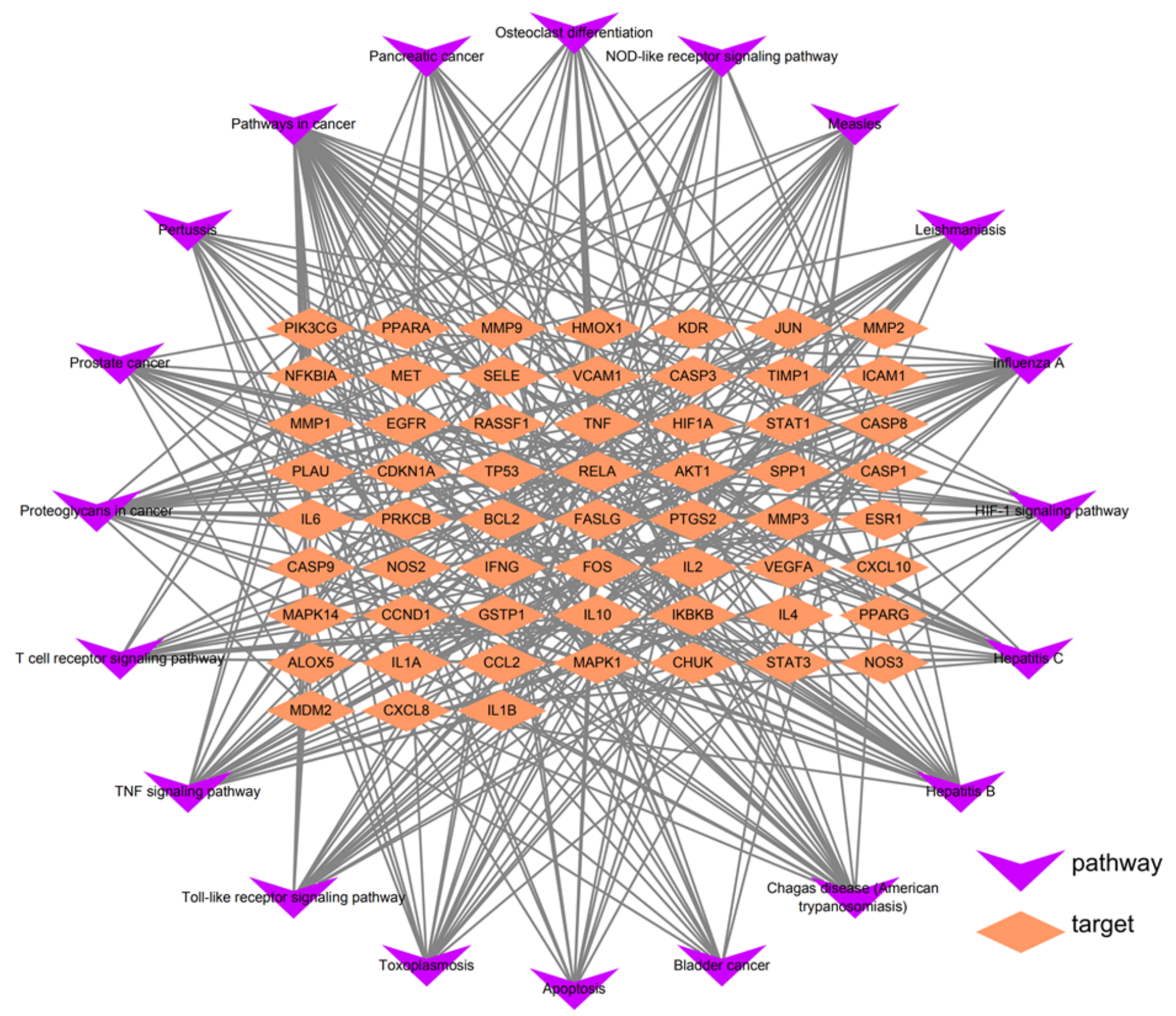

Figure 9

Targets-Pathways Network (T-P Network). 